\title{
Two Remarks on the Computer Study of Differentiable Dynamical Systems
}

\author{
Massimo Campanino* \\ Institut des Hautes Etudes Scientifiques, 35, Route de Chartres, F-91440 Bures-sur-Yvette, France
}

\begin{abstract}
In the first part of this note we find conditions under which the frequency spectrum of a transformation exhibits delta functions. In the second part we show that if an ergodic flow on an $m$-dimensional manifold has $m-1$ strictly negative characteristic exponents, then the measure is concentrated either on a fixed point or on a closed attracting orbit.
\end{abstract}

\section{Introduction}

In this note we examine some relations between the properties of an invariant measure (for a transformation or a flow) and some of its numerical characteristics, viz. the frequency spectrum and the characteristic exponents. Our remarks are relevant to studies by computer.

In Sect. 2 we find conditions under which the frequency spectrum of a transformation exhibits delta functions. A situation like the one we describe (cyclic permutation of attractors) arises in the case of the Hénon map for various values of the parameter (see [3] and [2]). In Sect. 3 we study an ergodic flow on a compact manifold. We show that if all the characteristic exponents but one are strictly negative, then all the measure is carried by a fixed point or by an attracting closed orbit. This generalizes the analogous result for transformations ([5], Corollary 6.2). For the basic definitions and results on transformations and flows see [1] and [4].

\section{Presence of Delta Functions in the Frequency Spectrum}

We consider a continuous transformation $T$ of a topological space. We assume that there is a finite number of disjoint compact sets which are cyclically permuted by $T$ and a Borel $T$ invariant measure, whose support is the union of the compacts. A standard method for studying the dynamical system generated by a transfor-

* Permanent address: Istituto Matematico "G. Castelnuovo", Universita degli Studi di Roma, Piazzale delle scienze, I-00100 Roma, Italy 
mation is to plot some real coordinate and to study its frequency spectrum. Our purpose is to find conditions, under which the frequency spectrum of some coordinate, with respect to $T$, exhibits delta functions in points different from 0 .

Let $\Omega$ be a topological space and $T$ a continuous map $T: \Omega \rightarrow \Omega$. We assume that there are $q$ compact subsets of $\Omega K_{0}, \ldots, K_{q-1}$ such that $K_{i} \cap K_{j} \neq \emptyset$ for $i \neq j$, $f\left(K_{i}\right) \subset K_{i+1}$ for $0 \leqq i \leqq q-2$ and $f\left(K_{q-1}\right) \subset K_{0}$ and that there is a $T$ invariant probability measure $\mu$ on $\Omega$ such that $\operatorname{supp} \mu=K=\bigcup_{l=0}^{q-1} K_{l}$.

We define:

$$
\mu_{l}=\left.q \mu\right|_{K_{l}} .
$$

Given a continuous function $\varphi: \Omega \rightarrow \mathbb{C}$, we can consider its correlation function $a_{\varphi}$ with respect to the measure $\mu$ and the map $T$ and the averages of $\varphi$ with respect to the measures $\mu_{l}$ :

$$
\begin{aligned}
a_{\varphi}(k) & =\int \varphi\left(T^{k} x\right) \bar{\varphi}(x) d \mu(x) \\
b_{\varphi}(l) & =\int \varphi(x) d \mu_{l}(x) .
\end{aligned}
$$

We can assume, without loss of generality that the map $T$ is invertible (see Remark 2.2). In this case the definition of $a_{\varphi}(k)$ makes sense for every $k \in \mathbb{Z}$ and we have:

$$
a_{\varphi}(-k)=\overline{a_{\varphi}(k)} \text {. }
$$

The sequence

$$
a_{\varphi}(k)=\int_{0^{-}}^{2 \pi^{-}} e^{i k \lambda} d F_{\varphi}(\lambda)
$$

where $d F_{\varphi}(\lambda)$ is a positive measure on the interval $[0,2 \pi)$.

We define $h_{\varphi}(n)=F\left(\frac{2 \pi n}{q}+\right)-F\left(\frac{2 \pi n}{q}-\right) n=0, \ldots, q-1$ and we put $G_{q}$ to be the subgroup of the group of the roots of the unity generated by the set $\left\{e^{\frac{i 2 \pi n}{q}} \mid h_{\varphi}(n)>0\right\}$. Then we have the following:

Theorem 2.1. Let $p$ be the smallest positive integer such that $b_{\varphi}(l)=b_{\varphi}(m)$ $\forall l, m \in\{0, \ldots, q-1\}$ with $l=m(\bmod p)$ and let $H_{\varphi}$ be the group $\left\{e^{\frac{i 2 \pi n}{p}} \mid 0 \leqq n \leqq p-1\right\}$. Then we have $H_{\varphi} \subset G_{\varphi}$. If $T$ is ergodic, then $H_{\varphi}=G_{\varphi}$.

Proof. Let $U$ be the unitary operator on $L_{2}(\Omega, \mu)$ induced by $T$

$$
U f(x)=f(T x) .
$$

$U$ can be written in terms of its spectral decomposition as:

$$
U=\int_{0^{-}}^{2 \pi^{-}} e^{i \lambda} d E(\lambda)
$$


We have:

$$
F_{\varphi}(\lambda)=(E(\lambda) \varphi, \varphi)
$$

where the scalar product $(\cdot, \cdot)$ is defined as

$$
(f, g)=\int f(x) \bar{g}(x) d \mu(x) .
$$

The functions :

$$
f_{n}(x)=\sum_{l=0}^{q-1} e^{\frac{i 2 \pi l n}{q}} \chi_{K_{l}}(x) \quad n=0,1, \ldots, q-1,
$$

where

$$
\chi_{K_{l}}(x)= \begin{cases}1 & x \in K_{l} \\ 0 & \text { otherwise }\end{cases}
$$

are eigenfunctions of $U$ corresponding respectively to the eigenvalues $e^{\frac{i 2 \pi n}{q}}$, as it follows from the properties of $T$. Therefore the space

$$
\left[E\left(\frac{2 \pi n}{q}+\right)-E\left(\frac{2 \pi n}{q}-\right)\right] L_{2}(\Omega, \mu)
$$

contains $f_{n}(x)$ and we have:

$$
h_{\varphi}(n)=\left(\left[E\left(\frac{2 \pi n}{q}+\right)-E\left(\frac{2 \pi n}{q}-\right)\right] \varphi, \varphi\right) \geqq\left|\left(f_{n}, \varphi\right)\right|^{2}=\left|c_{\varphi}(n)\right|^{2},
$$

where

$$
c_{\varphi}(n)=\frac{1}{q} \sum_{l=0}^{q-1} b_{\varphi}(l) e^{\frac{i 2 \pi n l}{q}}
$$

or equivalently

$$
b_{\varphi}(l)=\frac{1}{q} \sum_{l=0}^{q-1} c_{\varphi}(n) e^{\frac{-i 2 \pi l n}{q}} .
$$

If $T$ is $\mu$ ergodic, then all the eigenvalues of $U$ are simple (see e.g. [1]). So we have in this case:

$$
h_{\varphi}(n)=\left|c_{\varphi}(n)\right|^{2} \text {. }
$$

The theorem follows readily from (2.10), (2.11), and (2.12).

Remark 2.2. We note that if $T$ is not invertible, there is a standard way to construct an "equivalent" invertible $\tilde{T}$ acting on a space $\tilde{K}$. Let us consider the set

$$
\tilde{K}=\left\{\left(x_{n}\right) \in K^{\mathbb{Z}} \mid T\left(x_{n}\right)=x_{n+1}, \forall n \in \mathbb{Z}\right\}
$$

$\tilde{K}$ is a compact subset of $K^{\mathbb{Z}}$ with the product topology, since it is closed. Let $p_{0}$ be the projection $p_{0}: \tilde{K} \rightarrow \Omega$. 
$p_{0}\left(\left(x_{n}\right)\right)=x_{0}$ and let $\tilde{T}=\tilde{K} \rightarrow \tilde{K}$ be the shift $(\tilde{T} x)_{n}=x_{n+1}$. The following diagram is commutative and $\tilde{T}$ is invertible.

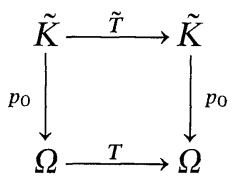

Let $\mu$ be a $T$ invariant measure on $\Omega$ with support on $K$. By Hahn-Banach and Markov-Kakutani theorems, there is a $\tilde{T}$ invariant probability measure on $\tilde{K}$ such that $p_{0}(\tilde{\mu})=\mu$. This measure is unique, because it is uniquely defined on the cylindrical sets.

So we can consider the space $\tilde{K}$, the invertible map $\tilde{T}$, the invariant measure $\tilde{\mu}$, the compact sets $\tilde{K}_{l}=p_{0}^{-1}\left(K_{l}\right)$, which are cyclically permuted by $\tilde{T}$, and the function $\tilde{\varphi}=\varphi \circ p_{0}$.

Remark 2.3. Let $\Omega=\mathbb{R}^{m}$ and assume that $q$ is a prime number. Assume also that some hyperplane separates two of the compact sets $K_{i}, K_{j}$ that is: $\exists t \in \mathbb{R}^{m}, u \in \mathbb{R}$ such that

$$
\begin{aligned}
& \sum_{n=1}^{m} t_{n} x_{n}>u \quad \forall x \in K_{i}, \\
& \sum_{n=1}^{m} t_{n} x_{n}<u \quad \forall x \in K_{j} .
\end{aligned}
$$

Then, of course

$$
\begin{aligned}
& \sum_{n=1}^{m} t_{n} b_{x_{n}}(i)>u, \\
& \sum_{n=1}^{m} t_{n} b_{x_{n}}(j)<u
\end{aligned}
$$

so that for some Cartesian coordinate $x_{n} 1 \leqq n \leqq m$ we have $b_{x_{n}}(i) \neq b_{x_{n}}(j)$, which implies, since $q$ is a prime number by Theorem 2.1 , that

$$
G_{x_{n}}=\left\{e^{\frac{i n \pi}{q}} \mid 0 \leqq n \leqq q-1\right\}
$$

If $q$ is not a prime number, we need that sufficiently many pairs of compact sets are separated by hyperplanes, in order to have that the group $G$ generated by $G_{x_{1}}, \ldots, G_{x_{m}}$ be equal to $\left\{e^{\frac{i 2 n \pi}{q}} \mid 0 \leqq n \leqq q-1\right\}$.

\section{Existence of an Attracting Closed Orbit}

Let $M$ be an $m$-dimensional compact differentiable manifold and let $f^{t}$ be a flow on it generated by a $C^{1+\alpha}$ vector field $X$. If $\varrho$ is an $f^{t}$ invariant probability measure on $M$, then the characteristic exponents are constant almost everywhere with respect to $\varrho$. We have the following result: 
Theorem 3.1. If the characteristic exponents of $f^{t}$ are all strictly negative, then supp $\varrho$ is an attractive fixed point for $f^{t}$. If $m-1$ characteristic exponents are strictly negative, then either (a) or (b) is verified:

(a) There is a fixed point $p$ of $f^{t}$ such that $\varrho(\{p\})=1$.

(b) There is an attracting closed orbit $\Delta$ for $f^{t}$ such that $\varrho(\Delta)=1$.

If $X(p) \neq 0$ for some point $p \in \operatorname{supp} \varrho$, then only (b) is possible.

Proof. The characteristic exponents for $f^{t}$ are the same as those for the $C^{1+\alpha}$ map $f^{1}$. Therefore the case where all the characteristic exponents are strictly negative follows from [5]. Let us now consider the case where $m-1$ characteristic exponents are strictly negative. In general there exists a Borel set $\Gamma \subset M$ such that $f^{t}(\Gamma) \subset \Gamma \forall t \geqq 0, \varrho(\Gamma)=1$ and the characteristic exponents are constant on $\Gamma$. In view of Theorem 6.1 of [5] $\forall \lambda$ negative bigger than all the negative characteristic exponents and $\forall p \in \Gamma$ there is a $C^{1+\alpha} m-1$-dimensional manifold $U_{p}^{\lambda}(\alpha(p))$ :

$$
U_{p}^{\lambda}(\alpha(p))=\left\{y \in \bar{B}(p, \alpha(p)) \mid d\left(f^{n}(y), f^{n}(p)\right) \leqq \beta(p) e^{n \lambda}, \forall n \geqq 0\right\},
$$

where $\alpha(p)$ and $\beta(p)$ are given strictly positive measurable functions on $\Gamma$.

If, for all $p \in \operatorname{supp} \varrho \cap \Gamma, X(p)=0$, then by the ergodicity of $\varrho$, there is a point $\bar{p} \in \Gamma$, such that $\varrho(\{\bar{p}\})=1$ and $\bar{p}$ is a fixed point for $f^{t}$.

Let us suppose that there exists a point $p \in \operatorname{supp} \varrho \cap \Gamma$ such that the vector field $X$ is different from 0 in $p$. The vector $X(p)$ is transversal to the manifold $U_{p}^{\lambda}(\alpha(p))$, since $U_{p}^{\lambda}(\alpha(p))$ is tangent in $p$ to $V_{p}^{\lambda}$, the subspace of $T_{p}(M)$ associated to all the negative exponents of $f^{t}$, whereas the vector $X(p)$ has 0 exponent, since it belongs to the field generating the flow.

Therefore there is a $\gamma>0$ and an open submanifold $U$ of $U_{p}^{\lambda}(\alpha(p))$ such that $p \in U$ and

$$
\psi: U \times(-\gamma, \gamma) \rightarrow M \quad \psi(x, t)=f^{t}(x)
$$

is a diffeomorphism of $U \times(-\gamma, \gamma)$ onto $\psi(U \times(-\gamma, \gamma))$.

Let $\bar{p}$ be a limit point for the sequence $\left(f^{n}(p)\right) n \geqq 0$ and let us consider the set $P=\left\{f^{t}(\bar{p}) \mid t \in\left[\frac{-\gamma}{2}, \frac{\gamma}{2}\right]\right\}$ and for $\varepsilon>0$ define:

$$
W_{\varepsilon}=\{r \in M \mid d(r, P)<\varepsilon\} .
$$

Given $\varepsilon>0$ we can find $\delta>0$ and integers $N \geqq 0$ and $n_{1} \geqq N$ such that:

$$
\begin{array}{ll}
\text { (i) } d\left(f^{t}(x), f^{t}(y)\right)<\varepsilon \quad \forall x, y \in M \quad & d(x, y)<\delta \quad|t| \leqq \frac{\gamma}{2} \\
\text { (ii) } d\left(f^{n}(x), f^{n}(p)\right)<\frac{\delta}{2} \quad \forall x \in U_{p}^{\lambda}(\alpha(p)) \quad \forall n \geqq N & \\
\text { (iii) } d\left(f^{n_{1}}(p), \bar{p}\right)<\frac{\delta}{2} . &
\end{array}
$$

We have $f^{n_{1}}\left(\psi\left(U \times\left[-\frac{\gamma}{2}, \frac{\gamma}{2}\right]\right)\right) \subset W_{\varepsilon}$. Let indeed $y \in f^{n_{1}}\left(\psi\left(U \times\left[-\frac{\gamma}{2}, \frac{\gamma}{2}\right]\right)\right)$, then $y=f^{t}\left(f^{n_{1}}(x)\right)$ with $x \in U$ and $|t| \leqq \frac{\gamma}{2}$. By using the inequalities (3.4) we obtain $d\left(y, f^{t}(\bar{p})\right)<\varepsilon$, which implies $y \in W_{\varepsilon}$. 
Therefore $\varrho\left(W_{\varepsilon}\right) \geqq \varrho\left(\psi\left(U \times\left[-\frac{\gamma}{2}, \frac{\gamma}{2}\right]\right)\right)=\mathcal{O}>0$, since $\psi\left(U \times\left[-\frac{\gamma}{2}, \frac{\gamma}{2}\right]\right)$ is a neighbourhood of $p$.

Since $\varepsilon>0$ is arbitrary.

$$
\varrho\left(W_{\varepsilon}\right) \geqq \mathcal{O} \quad \forall \varepsilon>0
$$

and

$$
\varrho(P)=\varrho\left(\bigcap_{n=1}^{\infty} W_{1 / n}\right)=\inf _{n} \varrho\left(W_{1 / n}\right) \geqq \mathcal{O}>0 .
$$

Therefore $P$ must be a recurrent set for a trajectory starting from $\bar{p}$, and two cases would be possible : either $P$ is part of a closed orbit $\Delta$, which is of course attracting, and $\varrho(\Delta)=1$, or else $\bar{p}$ is a fixed point with $\varrho(\{\bar{p}\})=1$. The latter case is actually not possible, since we could find a neighbourhood $V$ of $p$, such that $\bar{p} \notin V$, so that $\varrho(V)=0$, which contradicts $p \in \operatorname{supp} \varrho$.

Acknowledgements. I am deeply indebted to Prof. David Ruelle for his helpful advise and I acknowledge very kind hospitality at I.H.E.S.

\section{References}

1. Arnold, V.I., Avez, A.: Problèmes ergodiques de la mécanique classique. Paris: Gauthier Villars 1967

2. Curry, J.H.: On the Hénon transformation. Commun. Math. Phys. 68, 129-140 (1979)

3. Hénon, M.: A two dimensional mapping with a strange attractor. Commun. Math. Phys. 50, 69 (1976)

4. Ruelle, D.: Statistical mechanics and dynamical systems. Published by Mathematics Department Duke University 1976

5. Ruelle, D. : Ergodic theory of differentiable dynamical systems. Publications mathématiques I.H.E.S. No. 50 (1979)

Communicated by D. Ruelle

Received December 14, 1979 